\title{
Mutant DNA quantification by digital PCR can be confounded by heating during DNA fragmentation
}

\author{
Qing Kang1, Brian Parkin ${ }^{1}$, Maria D. Giraldez¹, and Muneesh Tewari ${ }^{1,2,3,4}$ \\ ${ }^{1}$ Department of Internal Medicine, Division of Hematology/Oncology, ${ }^{2}$ Department of Biomedical \\ Engineering, ${ }^{3}$ Center for Computational Medicine and Bioinformatics, and ${ }^{4}$ Biointerfaces Institute, \\ University of Michigan, Ann Arbor, MI
}

BioTechniques 60:175-185 (April 2016) doi 10.2144/000114401

Keywords: droplet digital PCR; DNA mutation; fragmentation; heating

Supplementary material for this article is available at www.BioTechniques.com/article/114401.

Digital PCR (dPCR) is gaining popularity as a DNA mutation quantification method for clinical specimens. Fragmentation prior to dPCR is required for non-fragmented genomic DNA samples; however, the effect of fragmentation on DNA analysis has not been well-studied. Here we evaluated three fragmentation methods for their effects on dPCR point mutation assay performance. Wild-type (WT) human genomic DNA was fragmented by heating, restriction digestion, or acoustic shearing using a Covaris focused-ultrasonicator. dPCR was then used to determine the limit of blank (LoB) by quantifying observed WT and mutant allele counts of the proto-oncogenes KRAS and BRAF in the WT DNA sample. DNA fragmentation by heating to $95^{\circ} \mathrm{C}$, while the simplest and least expensive method, produced a high background mutation frequency for certain KRAS mutations relative to the other methods. This was due to heat-induced mutations, specifically affecting dPCR assays designed to interrogate guanine to adenine ( $\mathrm{G}>\mathrm{A})$ mutations. Moreover, heat-induced fragmentation overestimated gene copy number, potentially due to denaturation and partition of single-stranded DNA into different droplets. Covaris acoustic shearing and restriction enzyme digestion showed similar LoBs and gene copy number estimates to one another. It should be noted that moderate heating, commonly used in genomic DNA extraction protocols, did not significantly increase observed KRAS mutation counts.

Absolute nucleic acid quantification using limiting dilution end-point PCR began in the 1990s, when this method was applied to detect HIV variants (1) and genetic rearrangements in acute lymphoblastic leukemia (AML) (2). After end point PCR, Poisson statistical analysis of the number of positive and negative reactions permitted calculation of the starting template concentration (3). This initial method was timeand resource-intensive because partitioning of PCR required large numbers of 96-well plates. Advances in nanofabrication and microfluidic technologies $(4,5)$ have made digital PCR (dPCR) routinely accessible through a number of platforms, including droplet-based instruments (e.g., Bio-Rad's Droplet Digital PCR System or Raindance Technologies' RainDrop Digital PCR System, which was used in this study). This technology has many applications, including detection of single nucleotide variants (SNVs), copy number variations (CNVs), and exogenous targets such as bacteria and viruses. One application for which dPCR has gained popularity is the detection of rare mutant alleles (6).

In dPCR, DNA concentration and size are determining factors in uniform droplet formation, because the viscosity of highly concentrated intact genomic DNA can change the average volume of droplets, which in turn could affect the accuracy of DNA quantitation (7). Therefore, fragmentation of intact genomic DNA samples and quality control of fragment sizes are critical pre-analytical steps in dPCR. Depending on the platform, DNA shearing has been recommended for input DNA concentrations $>66 \mathrm{ng} / 20 \mu \mathrm{L}$ reaction (using the Bio-Rad QX100/200 system) (4) or when using $>75 \mathrm{ng}$ of DNA per $25 \mu \mathrm{L}$ reaction (using the RainDrop system) (8). For the RainDrop system, it is recommended that DNA be fragmented to 3-4 kb to

\section{METHOD SUMMARY}

Wild-type (WT) human genomic DNA was fragmented by heating, restriction digestion, or acoustic shearing using a Covaris focused-ultrasonicator. Digital PCR (dPCR) was used to determine the limit of blank (LoB) by quantifying observed WT and mutant allele counts of the proto-oncogenes KRAS and BRAF in the WT DNA using a droplet-based dPCR system. 
ensure uniform droplet generation and amplifiable targets (8).

Current DNA fragmentation methods include double-strand breakage by heating (9), site-directed restriction enzyme digestion (4), digestion with an enzyme that generates random doublestrand breaks (e.g., Fragmentase) (10), acoustic shearing based on focusedultrasonication (e.g., using the Covaris focused-ultrasonicator), fragmentation with a biopolymer-based shredding system (QIAshredder, Qiagen) (11), and fragmenting by electromagnetic energy from microwave irradiation (12). Of these methods, high temperature is the simplest and least expensive approach. We adopted high temperature as a fragmentation method in developing dPCR assays for detecting SNVs using the RainDrop system, but found that heating had unanticipated effects on the results of DNA quantification: increased background mutation detection for certain alleles and greater than expected copy number quantification. These effects were not observed using either Covaris acoustic shearing or restriction enzyme digestion. We also found that moderate heating during genomic DNA extraction from whole blood did not cause an increase of false positive mutations, providing reassurance that the moderate heat incubation commonly used during DNA extraction is free from any significant mutagenic confounding effect. Here, we present a rationale for selecting an optimal DNA fragmentation strategy when performing $\mathrm{dPCR}$, especially for clinical research applications such as minimal residual disease (MRD) monitoring.

\section{Materials and methods}

Genomic DNA fragmentation

and quality control

Heating fragmentation was performed by incubating human genomic DNA (Promega, Madison, WI) at $95^{\circ} \mathrm{C}$ for 10 min, followed by pipetting 20 times using a $20-\mu \mathrm{L}$ pipette tip. The concentration of the DNA input was measured using a NanoDrop ND-1000 spectrometer (Wilmington, DE). Covaris acoustic shearing of $200 \mu \mathrm{L}$ genomic DNA (16 $\mu \mathrm{g}$ ) in blue miniTUBEs (Covaris, Woburn, MA), which generated DNA fragments centered on $3 \mathrm{~kb}$, was done using a S220 Focused-ultrasonicator (Covaris) with duty cycle $20 \%$, intensity 0.1 , and cycles per burst 1000, according to the manufacturer's instructions. Five units of restriction enzyme (Haelll or Msel) (0.5 $\mu \mathrm{L}$ ) (New England BioLabs, Ipswich, MA) was added to a $25-\mu \mathrm{L}$ dPCR (see below), unless otherwise stated, and incubated at room temperature for 10 min before applying the sample to a RainDrop source chip (RainDance Technologies, Billerica, MA) for droplet generation. DNA fragment sizes were analyzed using Genomic DNA ScreenTape on an Agilent 2200 TapeStation (Santa Clara, CA).

Primers, probes, and synthetic DNA fragments

Primers were obtained from IDT (Coralville, IA) with standard desalting purification. KRAS forward primer: 5'AAATGACTGAATATAAACTTGTGG-3'; KRAS reverse primer: 5'-TAGCTGTATCGTCAAGGCACTC-3'. It should be noted that it is possible that a KRAS pseudogene (KRASP1) could also be targeted by those primers; however, this would not change our conclusion that heat induces a higher false positive observed mutant frequency. Hydrolysis probes were synthesized by Life Technologies (Grand Island, NY) with wild-type (WT) and mutant sequences conjugated to VIC (Thermo Fisher Scientific, Waltham, MA) and 6-carboxyfluorescein (6-FAM) fluorophores, respectively. WT KRAS probe: 5'-TTGGAGCTGGTGGCGTA-3'; KRAS p.G12D (c. 35G>A) probe: 5'TTGGAGCTGATGGCGTA-3'; KRAS p.G12V (c. 35G>T) probe: 5' - TTGGAGCTGTTGGCGTA-3; KRAS p.G12S (c. 34G>A): 5'-TTGGAGCTAGTGGCGTA-3'. $B R A F$ primers and probe sequences are described elsewhere (4). Synthetic KRAS and BRAF mutant DNAs were synthesized as 225- or 200-bp gBlocks Gene Fragments by IDT with the SNV site located in the center of the synthetic gene sequence. The gBlock Gene Fragments (200 ng) were resuspended in TE buffer to a final concentration of $40 \mathrm{ng} / \mu \mathrm{L}$ in 5 $\mu \mathrm{L}$ aliquots. Aliquots were serially diluted with nuclease-free water to 4000 copies/ $\mu \mathrm{L}$, and $1 \mu \mathrm{L}$ was spiked into normal WT human genomic DNA in dPCR assays as a positive control.

\section{Droplet digital PCR}

A RainDrop Digital PCR System (RainDance Technologies) was used for detection of KRAS and BRAF mutations. In a pre-PCR environment, 1× TaqMan
Genotyping Master Mix (Life Technologies) was mixed with $900 \mathrm{nM}$ forward and reverse primers, $200 \mathrm{nM} 6$-FAM and VIC hydrolysis probes (Life Technologies), 1× Droplet Stabilizer (RainDance Technologies), and DNA template to a final volume of $25 \mu \mathrm{L}$ (unless stated otherwise) in 8-strip PCR tubes (Axygen, Tewksbury, MA). Reaction mixtures were pipetted into 8-well source chips (RainDance Technologies) and applied to a RainDrop Source instrument for droplet generation. Droplets were produced into new 8-strip PCR tubes (final volume $75 \mu \mathrm{L}$ ), sealed with opaque flat caps (RainDance Technologies), and PCR amplified to end point in a C1000 Touch thermal cycler with a 96-deep well reaction module (Bio-Rad, Hercules, CA). The thermal cycling conditions were: $10 \mathrm{~min}$ at $95^{\circ} \mathrm{C}$, followed by 45 cycles of $15 \mathrm{~s}$ at $95^{\circ} \mathrm{C}, 1$ min for annealing and extension (at $64^{\circ} \mathrm{C}$ for $K R A S$ and $60^{\circ} \mathrm{C}$ for BRAF assay, with $0.5^{\circ} \mathrm{C} / \mathrm{sec}$ ramp rate), followed by $10 \mathrm{~min}$ at $98^{\circ} \mathrm{C}$ and storage at $12^{\circ} \mathrm{C}$ or immediate application to a RainDrop Sense instrument for droplet analysis.

Droplet data analysis

Calculation of WT copy numbers in genome equivalents (GE) assumed a haploid genome mass equivalent of 3.23 $\mathrm{pg}$ and used the equation GE = DNA mass (pg) / 3.23 (pg). Due to droplet loss in the RainDrop Sense instrument, the number of measured target droplets was corrected using the equation: measured target droplets $=$ (total droplets generated / total intact droplets $) \times($ counted droplets with target of interest). The mutant DNA false-positive percentage was calculated from dPCR results obtained when using normal WT human genomic DNA without spiked mutant DNA as input, following the equation: false positive\% = (measured positive droplets in the mutant channel) / (measured positive droplets in both the WT and mutant channels) $\times 100 \%$. Limit of blank (LoB) was calculated with the formula $L o B=$ mean $_{\text {false positive } \%}+1.645 \times$ $\mathrm{SD}_{\text {false positive\% }}$ (12).

\section{Blood genomic DNA extraction}

Human whole blood was purchased from BioreclamationIVT (Westbury, NY). Whole blood was pooled from 3 healthy donors that included both men and women, preserved with $\mathrm{K}_{2}$ EDTA, and shipped at $4^{\circ} \mathrm{C}$ overnight. Whole blood was stored at $-80^{\circ} \mathrm{C}$ until DNA extraction. 
A

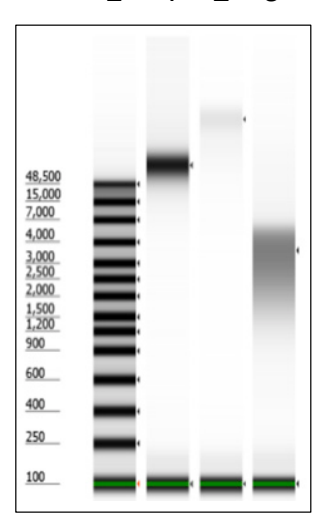

B
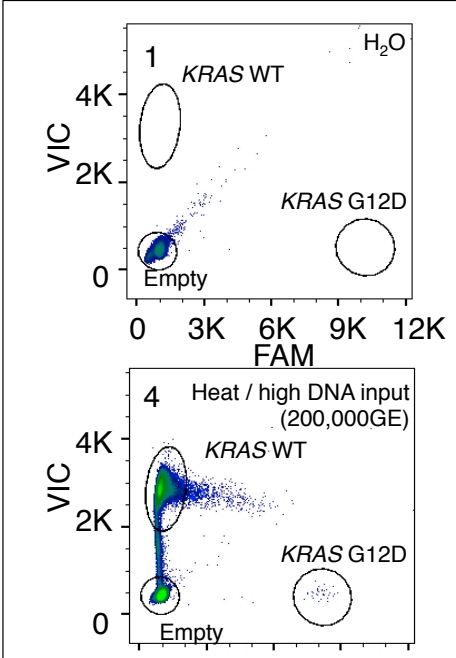

$0 \quad 3 \mathrm{~K} \underset{\text { FAM }}{6 \mathrm{~K}} 9 \mathrm{~K} \quad 12 \mathrm{~K}$
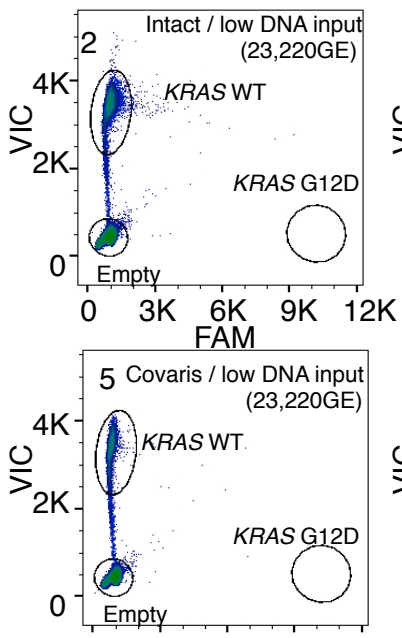

$0 \quad 3 \mathrm{~K} \underset{\text { FAM }}{\mathrm{KK}} 9 \mathrm{~K} \quad 12 \mathrm{~K}$
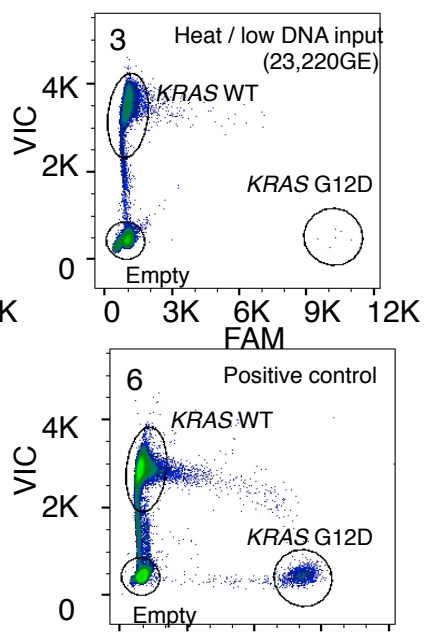

$0 \quad 3 \mathrm{~K} \quad 6 \mathrm{~K} \quad 9 \mathrm{~K} \quad 12 \mathrm{~K}$

\begin{tabular}{|c|c|c|c|c|c|c|c|c|c|}
\hline Panel & Assay & Sample & $\begin{array}{c}\text { \# Droplets } \\
\text { generated }\end{array}$ & $\begin{array}{c}\text { Total intact } \\
\text { droplets }\end{array}$ & $\begin{array}{c}\text { Measured } \\
\text { mutant } \\
\text { droplets }\end{array}$ & $\begin{array}{c}\text { Measured } \\
\text { WT droplets }\end{array}$ & $\begin{array}{c}\text { Predicted } \\
\text { WT copies }\end{array}$ & $\begin{array}{c}\text { Efficiency of } \\
\text { WT allele } \\
\text { recovery\% }\end{array}$ & $\begin{array}{c}\text { Mutant } \\
\text { false } \\
\text { positive\% }\end{array}$ \\
\hline 1 & $\begin{array}{c}\text { KRAS } \\
\text { G12D }\end{array}$ & $\mathrm{H}_{2} \mathrm{O}$ & $5,000,000$ & $3,443,529$ & 0 & 0 & 0 & $\mathrm{NA}$ & NA \\
\hline 2 & $\begin{array}{l}K R A S \\
\mathrm{G} 12 \mathrm{D}\end{array}$ & $\begin{array}{c}\text { Intact / low DNA } \\
\text { input }\end{array}$ & $5,000,000$ & $3,661,218$ & 0 & 20,639 & 23,220 & $88.9 \%$ & 0 \\
\hline 3 & $\begin{array}{l}K R A S \\
\mathrm{G} 12 \mathrm{D}\end{array}$ & $\begin{array}{c}\text { Heat / low DNA } \\
\text { input }\end{array}$ & $5,000,000$ & $3,602,638$ & 8 & 35,113 & 23,220 & $151.2 \%$ & $0.024 \%$ \\
\hline 4 & $\begin{array}{l}K R A S \\
\mathrm{G} 12 \mathrm{D}\end{array}$ & $\begin{array}{c}\text { Heat / high DNA } \\
\text { input }\end{array}$ & $5,000,000$ & $4,149,031$ & 41 & 343,037 & 200,000 & $171.5 \%$ & $0.012 \%$ \\
\hline 5 & $\begin{array}{l}K R A S \\
\mathrm{G} 12 \mathrm{D}\end{array}$ & $\begin{array}{c}\text { Covaris / low DNA } \\
\text { input }\end{array}$ & $5,000,000$ & $3,445,162$ & 0 & 21,706 & 23,220 & $93.5 \%$ & 0 \\
\hline 6 & $\begin{array}{c}K R A S \\
\mathrm{G} 12 \mathrm{D}\end{array}$ & $\begin{array}{c}\text { Heat / high DNA } \\
\text { input +KRAS G12D } \\
\text { (positive control) }\end{array}$ & $5,000,000$ & $3,905,957$ & 1,866 & 303,751 & 200,000 & $151.9 \%$ & NA \\
\hline
\end{tabular}

Figure 1. Heat fragmentation increases false positives of KRAS G12D (c.35G>A) in digital (dPCR) analysis. (A) Intact (Lane 1), heat-fragmented (Lane 2), and Covaris acoustically sheared (Lane 3) normal human genomic DNA obtained on an Agilent TapeStation 2200 instrument specifically detecting doublestranded DNA. Heat-fragmented DNA showed a decreased overall signal and was larger, which may be due to heat-induced melting of the genomic DNA to single-strand DNA with partial re-annealing of repetitive sequences resulting in higher molecular weight complexes. L: ladder. (B) KRAS G12D dPCR data using heat-fragmented or Covaris acoustically sheared genomic DNA. Panel 1: no template; Panel 2: intact genomic DNA, low DNA input (23,220 GE); Panel 3: heat-fragmented genomic DNA, low DNA input; Panel 4: heat-fragmented genomic DNA, high DNA input (200,000 GE); Panel 5: Covaris acoustically sheared genomic DNA, low DNA input; Panel 6: positive control, synthetic KRAS G12D DNA fragments spiked into heat-fragmented genomic DNA. GE: genome equivalents. (C) Tabulated dPCR results for the conditions described in (B). Predicted wild-type (WT) copies = Input DNA mass (pg) / 3.23 (pg), where 1 haploid human genome is estimated as 3.23 pg. Efficiency of WT allele recovery\% = measured WT droplets / predicted WT copies $\times 100 \%$. Mutant false positive $\%=$ measured mutant droplets $/$ (measured WT droplets + measured mutant droplets) $\times 100 \%$. NA: not applicable.

Blood genomic DNA was extracted from $200 \mu \mathrm{L}$ whole blood using the QIAamp DNA Blood Mini Kit (Qiagen, Valencia, CA). Purification protocols were modified from the manufacturer's instructions to test the effects of temperature during protease incubation and RNase treatment on dPCR performance: (i) Blood lysates were incubated at room temperature, $56^{\circ} \mathrm{C}$, or $70^{\circ} \mathrm{C}$ for $10 \mathrm{~min}$ after supplementing with $20 \mu \mathrm{L}$ Qiagen Protease and $200 \mu \mathrm{L}$ AL buffer. (ii) At the elution step, columns were eluted in $50 \mu \mathrm{L} \mathrm{AE}$ buffer with or without $0.4 \mu \mathrm{L}$ RNase $\mathrm{A}$ solution (Promega) and incubated at room temperature for 10 min after elution. DNA concentration was determined using a NanoDrop ND-1000 spectrophotometer and the Qubit dsDNA HS Assay Kit (Life Technologies). Isolated DNA was aliquoted and stored at $-20^{\circ} \mathrm{C}$ until dPCR analysis.

\section{Results and discussion}

The LoB and the limit of detection (LoD) are part of the digital MIQE (Minimum Information for publication of Quanti- tative dPCR Experiments) (13). The LoB determines the LoD (the lowest concentration of analyte that can be reliably detected above the LoB) and thus is a critical parameter for defining the sensitivity of rare mutant allele detection in a WT background. LoB is defined by the frequency of positive droplets detected in samples with no target DNA present (14). To determine the LoB of the dPCR assay for detecting KRAS G12D (c.35G>A), a common oncogenic mutation, we analyzed normal, WT human genomic DNA that had been 
A
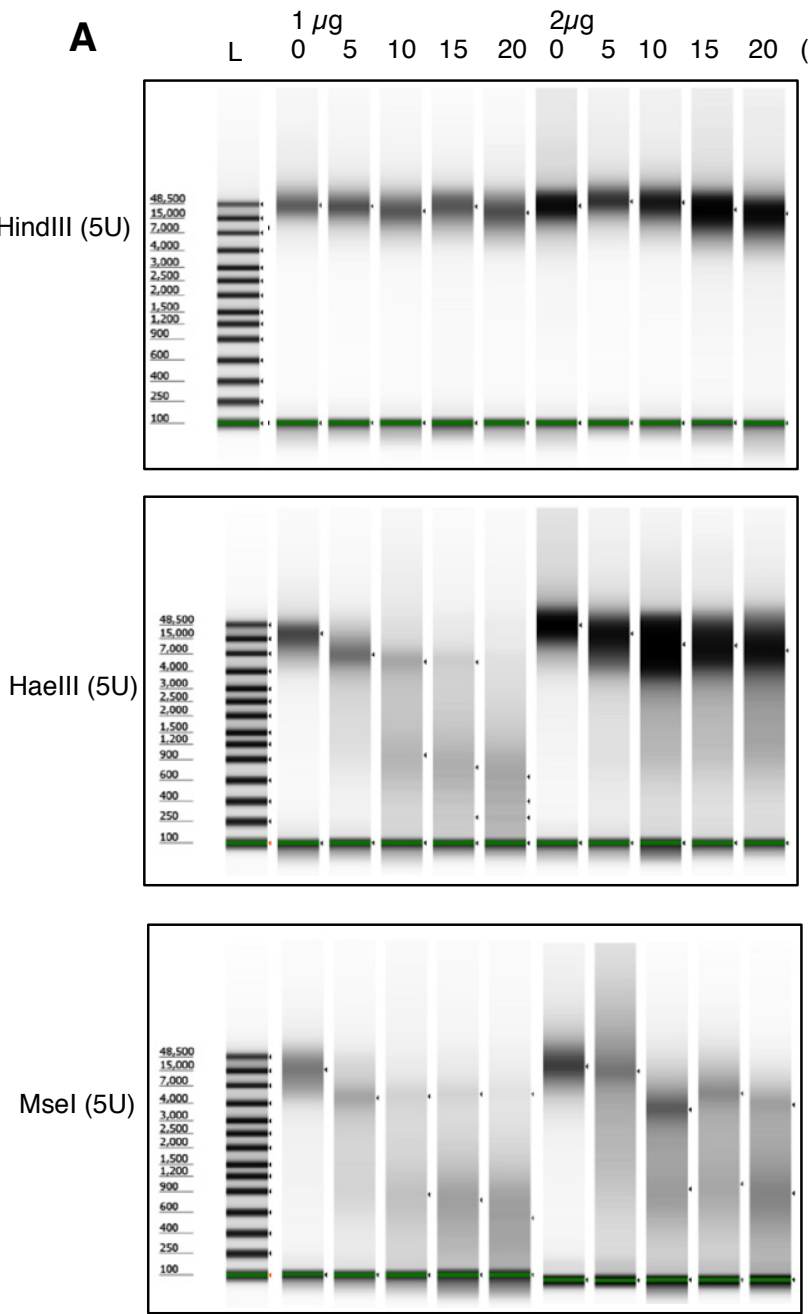

\section{B}

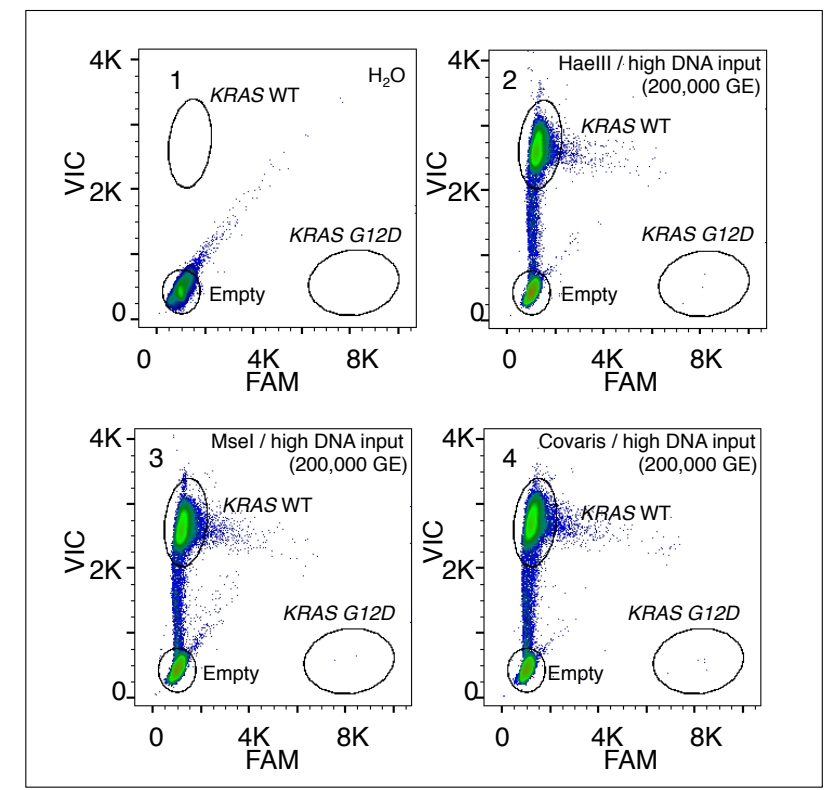

C

\begin{tabular}{|c|c|c|c|}
\hline $\begin{array}{c}\text { Measured } \\
\text { Parameters }\end{array}$ & Covaris & Haell & Msel \\
\hline $\begin{array}{c}\text { Mutant false } \\
\text { positive } \pm \text { SD } \%\end{array}$ & $\begin{array}{c}0.00276 \% \pm \\
0.00106 \%\end{array}$ & $\begin{array}{c}0.00227 \% \pm \\
0.00086 \%\end{array}$ & $\begin{array}{c}0.00230 \% \pm \\
0.00214 \%\end{array}$ \\
\hline LoB & $0.00451 \%$ & $0.00368 \%$ & $0.00582 \%$ \\
\hline $\begin{array}{c}\text { Efficiency of WT } \\
\text { allele recovery } \%\end{array}$ & $92.7 \% \pm 1.2 \%$ & $88.7 \% \pm 4.5 \%$ & $89.1 \% \pm 4.1 \%$ \\
\hline
\end{tabular}

Figure 2. Restriction enzyme-digested and Covaris acoustically sheared genomic DNA show similar limits of blank (LoBs) for KRASG12D (c.35G>A) assays. (A) Agilent TapeStation 2200 images of wild-type (WT) genomic DNA digested for varying times with HindIII-, HaellI-, or Msel. (B) KRAS G12D digital PCR (dPCR) data generated using Haelll- or Msel-digested or Covaris acoustically sheared genomic DNA samples (200,000 GE). GE: genome equivalents. Samples were analyzed as in Figure 1. VIC, excitation $\bigotimes: 528$ nm, emission 囚: 554 nm. 6-carboxyfluorescein (FAM), excitation $\otimes: 494 \mathrm{~nm}$. emission : $522 \mathrm{~nm}$. The $\mathrm{x}$-axis and $\mathrm{y}$-axis represent fluorescence units observed for FAM and VIC, respectively. (C) Table comparing KRAS G12D (c.35G>A) dPCR assay results from Haelll- or Msel-digested or Covaris acoustically sheared genomic DNA. Results represent mean and SD of data from $5 \mathrm{dPCR}$ replicates for each fragmentation method. $\mathrm{LoB}=$ mean $_{\text {mutant false positive }}+1.645 \times\left(\mathrm{SD}_{\text {mutant false positive }}\right)$.

fragmented by heating to $95^{\circ} \mathrm{C}$ for 10 min. In dPCR assays for KRAS G12D using $75 \mathrm{ng}$ of unfragmented input DNA (23,220 GE), zero mutant copies were detected (Figure 1, B and C, Panel 2). However, when using heat-fragmented DNA, the assay detected 8 copies of mutant DNA, with a fractional abundance of $0.024 \%$ for mutant alleles (Figure 1, B and $\mathrm{C}$, Panel 3). When total DNA input was increased to 200,000 GE (646 ng), detection of mutant copies increased to 41 , with a fractional abundance of $0.012 \%$ for mutant alleles (Figure 1, $B$ and $C$, Panel 4). We next evaluated the same mutation using WT genomic DNA fragmented by Covaris acoustic shearing, which generated a range of DNA fragment sizes, with a peak at 3-4 kb (Figure 1A, Lane 3; Supplementary Figure S1, Lanes 3 and 4). Using 23,220 GE of Covaris acoustically sheared WT genomic DNA, the $\mathrm{dPCR}$ assay detected zero mutant copies, a result similar to intact DNA (Figure 1, B and C, Panel 5). At $200,000 \mathrm{GE}$ of sheared input DNA, 5 mutant copies were detected, representing a fractional abundance of $0.00276 \%$ for mutant alleles (Figure 2B, Panel 4 and Figure 2C).

One possible explanation for the observed increase of LoB in KRAS G12D (c. $35 \mathrm{G}>\mathrm{A}$ ) assays when using heating for DNA fragmentation is the appearance of new heat-induced mutations. Heating at high temperature causes deamination of cytosine $(C)$ to uracil $(U)$ in WT DNA (15), which might enable the
PCR probe that detected the mutant DNA with thymine $(T)$ (complementary to A) to recognize a heat-induced mutation site containing $U$. To test this hypothesis, we set up additional KRAS mutation assays targeting KRAS G12V (c.35G>T) and KRAS G12S (c.34G>A). The only difference among the three $K R A S$ mutation assays was in a single nucleotide in the probe that detected the point mutation site. Using heatfragmented genomic DNA $(200,000$ GE), we observed zero false positives in the KRAS G12V assay detecting a $\mathrm{G}>\mathrm{T}$ mutation, but 50 false positives (representing a fractional abundance of $0.017 \%$ ) in the KRAS G12S assay detecting a $G>A$ mutation, similar to results from the KRAS G12D assay, which also targeted 


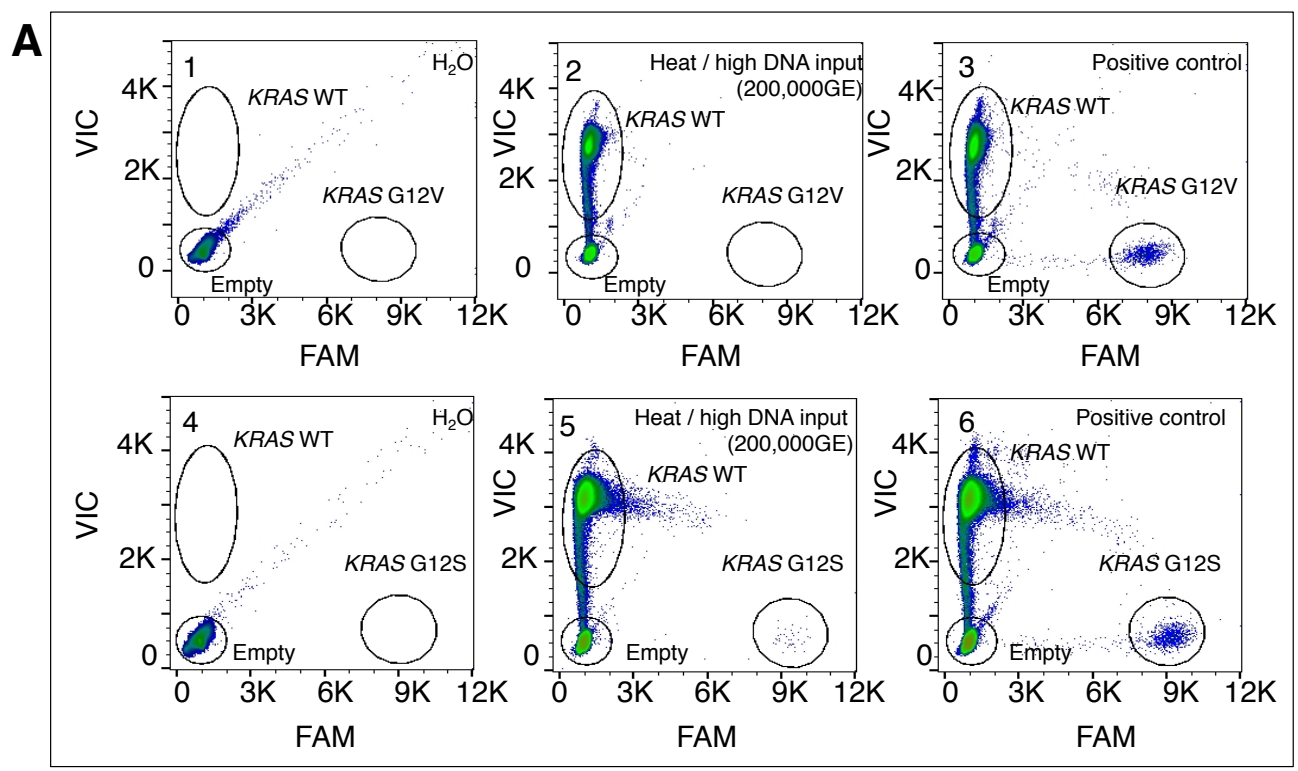

\begin{tabular}{|c|c|c|c|c|c|c|c|c|c|}
\hline Panel & Assay & Sample & $\begin{array}{l}\text { \# droplets } \\
\text { generated }\end{array}$ & $\begin{array}{l}\text { Total intact } \\
\text { droplets }\end{array}$ & $\begin{array}{c}\text { Measured } \\
\text { mutant droplets }\end{array}$ & $\begin{array}{l}\text { Measured } \\
\text { WT droplets }\end{array}$ & $\begin{array}{c}\text { Predicted } \\
\text { copies WT }\end{array}$ & $\begin{array}{l}\text { Efficiency of } \\
\text { WT allele } \\
\text { recovery \% } \\
\end{array}$ & \begin{tabular}{|c|} 
Mutant \\
false \\
positive $\%$ \\
\end{tabular} \\
\hline 1 & $\begin{array}{l}\text { KRAS } \\
\text { G12V }\end{array}$ & $\mathrm{H}_{2} \mathrm{O}$ & $5,000,000$ & $3,136,875$ & 0 & 0 & 0 & (2) & NA \\
\hline 2 & $\begin{array}{l}\text { KRAS } \\
\text { G12V }\end{array}$ & $\begin{array}{c}\text { Heat / high DNA } \\
\text { input }\end{array}$ & $5,000,000$ & $3,749,021$ & 0 & 315,973 & 200,000 & $158.0 \%$ & 0 \\
\hline 3 & $\begin{array}{l}\text { KRAS } \\
\text { G12V }\end{array}$ & $\begin{array}{c}\text { Heat / high DNA } \\
\text { input }+K R A S \\
\text { G12V } \\
\text { (positive control) }\end{array}$ & $5,000,000$ & $4,046,695$ & 1,133 & 320,349 & 200,000 & $160.2 \%$ & NA \\
\hline 4 & $\begin{array}{l}\text { KRAS } \\
\text { G12S }\end{array}$ & $\mathrm{H} 2 \mathrm{O}$ & $5,000,000$ & $3,152,685$ & 0 & 0 & 0 & 0 & NA \\
\hline 5 & $\begin{array}{l}\text { KRAS } \\
\text { G12S }\end{array}$ & $\begin{array}{c}\text { Heat / high DNA } \\
\text { input }\end{array}$ & $5,000,000$ & $4,083,349$ & 50 & 297,626 & 200,000 & $148.8 \%$ & $0.017 \%$ \\
\hline 6 & $\begin{array}{l}\text { KRAS } \\
\text { G12S }\end{array}$ & $\begin{array}{c}\text { Heat / high DNA } \\
\text { input }+ \text { KRAS } \\
\text { G12S } \\
\text { (positive control) }\end{array}$ & $5,000,000$ & $4,074,023$ & 1,049 & 302,626 & 200,000 & $151.3 \%$ & NA \\
\hline
\end{tabular}

Figure 3. Heat fragmentation increases false positives in digital PCR (dPCR) assays for G>A (KRAS G12S) but not G>T (KRAS G12V). (A) dPCR data from KRAS G12V (c.35G>T) and KRAS G12S (c.34G>A) assays applied to heat-fragmented genomic DNA. Panel 1: no template; Panel 2: heat-fragmented genomic DNA (200,000 GE); Panel 3: synthetic KRAS G12V DNA spiked into heat-fragmented genomic DNA (200,000 GE); Panel 4: no template; Panel 5, heat-fragmented genomic DNA (200,000 GE); Panel 6: synthetic KRAS G12S DNA spiked into heat-fragmented genomic DNA (200,000 GE). GE: genome equivalents. (B) Tabulated dPCR results for the conditions described in (A). NA: not applicable.

a $\mathrm{G}>\mathrm{A}$ mutation (Figure $3, \mathrm{~A}$ and $\mathrm{B}$, Panels 2 and 5). In another dPCR assay detecting a non-G>A mutation, BRAF V600E (c.1799 T>A), heat-fragmented DNA gave zero false positives at 200,000 GE and only 1 mutant copy $(0.00032 \%$ fractional abundance) measured in 400,000 GE (Supplementary Figure S2, Panels 2 and 3 ). These results supported the hypothesis that heat induces DNA mutations specifically at $\mathrm{G} / \mathrm{C}$ sites and therefore increases the LoB for $G>A$ SNVs, but not for $\mathrm{G}>\mathrm{T}$ or $\mathrm{T}>\mathrm{A}$.

We also examined the efficiency of target DNA recovery, comparing the WT allele copy number measured by
dPCR with the expected input of WT DNA. Surprisingly, we found that heatfragmented genomic DNA demonstrated a recovery efficiency ranging 148.8\%-171.5\% (Figure 1C, Panels 3, 4, and 6; Figure 3B, Panels 2, 3, 5, and 6), suggesting an over-estimation of WT DNA copy number. In contrast, both intact and Covaris acoustically sheared genomic DNA showed an amplification efficiency $<100 \%$, as expected, ranging 88.9\%-93.5\% (Figure 1, B and C, Panels 2 and 5). This result might be explained by heat denaturation of double-stranded DNA to single-stranded DNA with insufficient re-annealing to complementary strands prior to incorporation into droplets for PCR. Initially, our result would seem to contradict a previous study using the Fluidigm BioMark system that reported heating can reduce the measured copy number of plasmid DNA by impairing DNA integrity (16). However, such an effect was only observed with heating to $95^{\circ} \mathrm{C}$ for $>10 \mathrm{~min}$, whereas with shorter heating times (30 s and 1 min), the measured copy number was increased compared with no heating (16). This is similar to our results but occurred on a different time scale, which may be explained by the reduced complexity of plasmid DNA in the prior study compared 
A

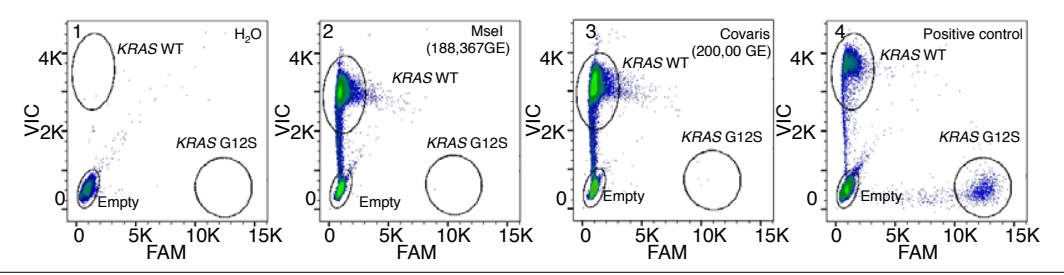

B

\begin{tabular}{|c|c|c|c|c|c|c|c|c|c|}
\hline Panel & Assay & Sample & $\begin{array}{c}\text { \# droplets } \\
\text { generated }\end{array}$ & $\begin{array}{c}\text { Total intact } \\
\text { droplets }\end{array}$ & $\begin{array}{c}\text { Measured } \\
\text { mutant droplets }\end{array}$ & $\begin{array}{c}\text { Measured } \\
\text { WT droplets }\end{array}$ & $\begin{array}{c}\text { Predicted } \\
\text { WT copies }\end{array}$ & $\begin{array}{c}\text { Efficiency of } \\
\text { WT allele } \\
\text { recovery\% }\end{array}$ & $\begin{array}{c}\text { Mutant } \\
\text { false } \\
\text { positive\% }\end{array}$ \\
\hline 1 & $\begin{array}{c}\text { KRAS } \\
\text { G12S }\end{array}$ & $\mathrm{H}_{2} \mathrm{O}$ & $5,000,000$ & $3,480,061$ & 0 & 3 & 0 & NA & NA \\
\hline 2 & $\begin{array}{l}\text { KRAS } \\
\text { G12S }\end{array}$ & Msel & $5,000,000$ & $3,974,052$ & 1 & 169,526 & 188,367 & $90.0 \%$ & $0.00074 \%$ \\
\hline 3 & $\begin{array}{c}\text { KRAS } \\
\text { G12S }\end{array}$ & Covaris & $5,000,000$ & $3,372,521$ & 6 & 178,782 & 200,000 & $89.4 \%$ & $0.00332 \%$ \\
\hline 4 & $\begin{array}{l}\text { KRAS } \\
\text { G12S }\end{array}$ & $\begin{array}{c}\text { Intact+KRAS } \\
\text { G12S } \\
\text { (positive control) }\end{array}$ & $5,000,000$ & $3,550,602$ & 1,331 & 13,089 & 14,570 & $89.8 \%$ & NA \\
\hline
\end{tabular}

C
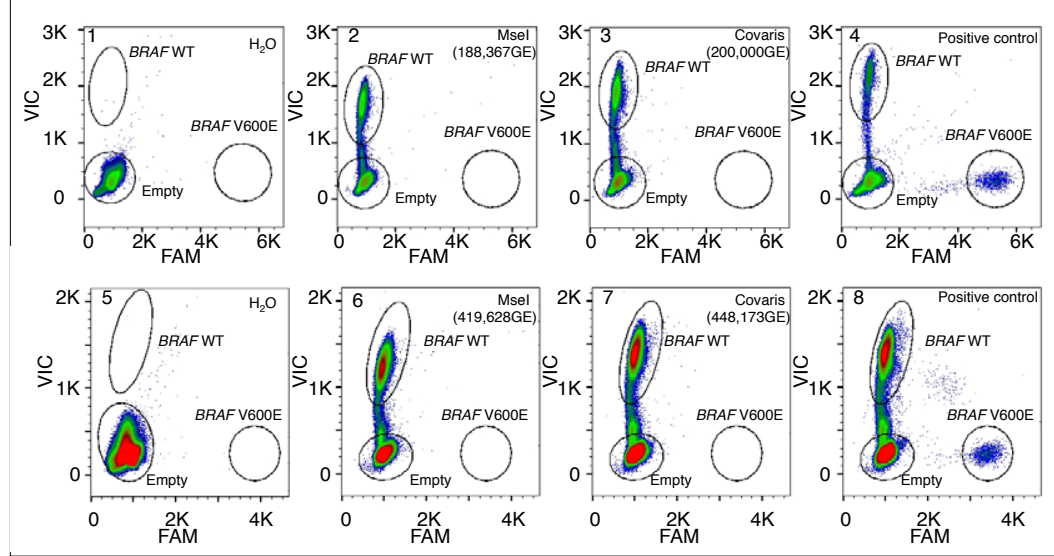

D

\begin{tabular}{|c|c|c|c|c|c|c|c|c|c|}
\hline Panel & Assay & Sample & $\begin{array}{c}\text { \# Droplets } \\
\text { generated }\end{array}$ & $\begin{array}{c}\text { Total intact } \\
\text { droplets }\end{array}$ & $\begin{array}{c}\text { Measured } \\
\text { Mutant droplets }\end{array}$ & $\begin{array}{c}\text { Measured } \\
\text { WT droplets }\end{array}$ & $\begin{array}{c}\text { Predicted } \\
\text { WT copies }\end{array}$ & $\begin{array}{c}\text { Efficiency of } \\
\text { WT allele } \\
\text { recovery } \%\end{array}$ & $\begin{array}{c}\text { Mutant } \\
\text { false } \\
\text { positive } \\
\%\end{array}$ \\
\hline 1 & $\begin{array}{c}B R A F \\
\text { V600E }\end{array}$ & $\mathrm{H}_{2} \mathrm{O}$ & $5,000,000$ & $3,678,801$ & 0 & 4 & 0 & $\mathrm{NA}$ & NA \\
\hline 2 & $\begin{array}{c}B R A F \\
\mathrm{~V} 600 \mathrm{E}\end{array}$ & Msel & $5,000,000$ & $3,947,653$ & 0 & 181,767 & 188,367 & $96.5 \%$ & $0 \%$ \\
\hline 3 & $\begin{array}{c}B R A F \\
\mathrm{~V} 600 \mathrm{E}\end{array}$ & Covaris & $5,000,000$ & $4,027,968$ & 0 & 184,125 & 200,000 & $92.1 \%$ & $0 \%$ \\
\hline 4 & $\begin{array}{c}B R A F \\
\mathrm{~V} 600 \mathrm{E}\end{array}$ & $\begin{array}{c}\text { Covaris+BRAF V600E } \\
\text { (positive control) }\end{array}$ & $5,000,000$ & $3,545,251$ & 1,967 & 13,610 & 14,490 & $93.9 \%$ & $\mathrm{NA}$ \\
\hline 5 & $\begin{array}{c}B R A F \\
\mathrm{~V} 600 \mathrm{E}\end{array}$ & $\mathrm{H} 2 \mathrm{O}$ & $5,000,000$ & $2,964,881$ & 0 & 2 & 0 & $\mathrm{NA}$ & $\mathrm{NA}$ \\
\hline 6 & $\begin{array}{c}B R A F \\
\mathrm{~V} 600 \mathrm{E}\end{array}$ & Msel & $5,000,000$ & $3,873,769$ & 0 & 398,115 & 419,628 & $94.9 \%$ & $0 \%$ \\
\hline 7 & $\begin{array}{c}B R A F \\
\mathrm{~V} 600 \mathrm{E}\end{array}$ & Covaris & $5,000,000$ & $3,740,580$ & 0 & 394,139 & 448,173 & $87.9 \%$ & $0 \%$ \\
\hline 8 & $\begin{array}{c}B R A F \\
\mathrm{~V} 600 \mathrm{E}\end{array}$ & $\begin{array}{c}\text { Covaris+BRAF V600E } \\
\text { (positive control) }\end{array}$ & $5,000,000$ & $3,944,471$ & 2,095 & 397,439 & 448,173 & $88.7 \%$ & NA \\
\hline
\end{tabular}

Figure 4. Restriction enzyme digestion and Covaris acoustic shearing of DNA yields similar results in KRAS G12S and BRAF V600E assays. (A) Digital PCR (dPCR) data from KRAS G12S (c.34G $>$ A) generated using Msel-digested or Covaris acoustically sheared genomic DNA as input. Panel 1: negative control, $\mathrm{H}_{2} \mathrm{O}$ (no template control); Panel 2: Msel-digested genomic DNA (188,367 GE) (10 min digestion at room temperature); Panel 3: Covaris acoustically sheared genomic DNA (200,000 GE); Panel 4: synthetic KRAS G12S DNA fragment spiked into intact genomic DNA (14,570 GE). GE: genome equivalents. (B) Tabulated dPCR results for the conditions described in (A). (C) dPCR data from BRAF V600E (c.1799T>A) assays using Mseldigested or Covaris acoustically sheared genomic DNA: no template (Panels 1 nd 5); Mseldigested genomic DNA (Panel 2, 188,367 GE; Panel 6, 419,628 GE); Covaris acoustically sheared genomic DNA (Panel 3, 200,000 GE; Panel 7, 448,173 GE); positive control, synthetic BRAF V600E DNA spiked into Covaris acoustically sheared genomic DNA (Panel 4, 14,490 GE; Panel 8, $448,173 \mathrm{GE}$ ). (D) Tabulated dPCR results for the conditions described in (C). NA: not applicable. with the human genomic DNA analyzed in our study.

Our results suggested that DNA fragmentation by heating increases LoB and copy number quantification and is thus not ideal as a shearing strategy for $\mathrm{dPCR}$, especially for assays measuring $G>A$ mutations. Restriction enzyme digestion is an alternative DNA fragmentation method recommended for the Bio-Rad QX platform, but it has not been well-studied for the RainDrop dPCR system (4). We tested the fragmentation efficiency of 3 commonly used restriction enzymes by performing the digestions in $1 \times$ PCR master mix at room temperature for $10 \mathrm{~min}$. The addition of $5 \mathrm{U}$ of Hindlll did not efficiently digest 1 or $2 \mu \mathrm{g}$ of genomic DNA to 3-4 kb (Figure 2A). In contrast, the addition of $5 \mathrm{U}$ of Haelll efficiently digested $1 \mu \mathrm{g}$ of genomic DNA to $\sim 4 \mathrm{~kb}$ or smaller; however, the efficiency of Haelll dropped substantially for $2 \mu \mathrm{g}$ DNA. Finally, treatment with $5 \mathrm{U}$ of Msel efficiently digested both 1 and $2 \mu \mathrm{g}$ of genomic DNA to $<4 \mathrm{~kb}$. Based on these results, we selected Haelll and Msel for further testing in both KRAS and $B R A F \mathrm{dPCR}$ assays after confirming that neither our targets nor our reference amplicons have Haelll or Msel recognition sites and that both enzymes are insensitive to methylation.

We analyzed the KRAS G12D mutation in the RainDrop dPCR system using WT genomic DNA fragmented by Covaris acoustic shearing or restriction enzyme digestion by Haell or Msel as input, with five replicates for each condition. The LoB and efficiency of recovery of target DNA were generally similar for all three fragmentation methods (Figure 2, B and C; Supplementary Figure S3) but modestly lower in the restriction enzyme-treated samples (i.e., $88.7 \%$ and $89.1 \%$ for restriction enzymes Haelll and Msel, respectively, versus $92.7 \%$ for Covaris acoustic shearing). This could potentially be due to PCR inhibition by the restriction enzyme storage buffer since we added the restriction enzyme directly to the sample without further purification prior to PCR.

We then compared the false-positive rates when using restriction enzymedigested DNA as input (using Msel because of its greater robustness to higher input of genomic DNA) versus Covaris acoustically sheared DNA 
Table 1. Limit of blank (LoB) of KRAS G12D (c.35G>A) droplet digital PCR (dPCR) assay using whole blood genomic DNA template isolated without RNase treatment.

\begin{tabular}{|c|c|c|c|c|c|c|}
\hline \multirow{2}{*}{ No RNase A } & \multicolumn{3}{|c|}{ KRAS G12D false positive \% } & \multicolumn{3}{|c|}{$\%$ efficiency of WT allele recovery } \\
\hline & RT & $56^{\circ} \mathrm{C}$ & $70^{\circ} \mathrm{C}$ & RT & $56^{\circ} \mathrm{C}$ & $70^{\circ} \mathrm{C}$ \\
\hline Extraction 1_replicate 1 & $0.00121 \%$ & $0.00098 \%$ & $0.00196 \%$ & $58.2 \%$ & $68.4 \%$ & $65.1 \%$ \\
\hline Extraction 1_replicate 2 & 0 & 0 & 0 & $55.6 \%$ & $67.0 \%$ & $64.7 \%$ \\
\hline Extraction 2_replicate 1 & $0.00099 \%$ & 0 & 0 & $68.8 \%$ & $65.5 \%$ & $51.3 \%$ \\
\hline Extraction 2_replicate 2 & $0.00094 \%$ & 0 & 0 & $67.8 \%$ & $64.0 \%$ & $50.9 \%$ \\
\hline Mean $(n=4)$ & $0.00078 \%$ & $0.00024 \%$ & $0.00049 \%$ & $62.6 \%$ & $66.2 \%$ & $58.0 \%$ \\
\hline SD & $0.00054 \%$ & $0.00049 \%$ & $0.00098 \%$ & $6.65 \%$ & $1.93 \%$ & $7.99 \%$ \\
\hline LoB & $0.00167 \%$ & $0.00105 \%$ & $0.00210 \%$ & & & \\
\hline
\end{tabular}

Whole blood genomic DNA template was isolated at varying temperatures without RNase treatment and then measured by a NanoDrop spectrophotometer. dPCR reaction volume: $30 \mu \mathrm{L}$. DNA input: 200,000 genome equivalents (GE) / reaction digested with $0.5 \mu \mathrm{L}(5 \mathrm{U})$ Msel for $10 \mathrm{~min}$ at room temperature (RT). WT: wild-type.
(Supplementary Figure S1). Mseldigested and Covaris acoustically sheared DNA showed similar falsepositive rates and efficiencies of input target recovery for KRAS G12S assays (Figure 4, A and B, Panels 2 and 3). The performance was also similar for BRAF V600E assays, with no false positives detected in either Msel-digested or Covaris acoustically sheared genomic DNA (Figure 4, C and D, Panels 2, 3, 6 , and 7 ). This result suggests that restriction enzyme digestion can be a simple and cost-effective alternative to Covaris acoustic shearing for dPCR assays using the RainDrop system. It is worth noting, however, that enzymes other than Haelll or Msel may need to be chosen depending on the target mutation(s) being analyzed.

An important application of dPCR using genomic DNA is MRD monitoring in hematopoietic disorders such as AML. DNA extraction for MRD analysis commonly requires incubating blood cell lysates with protease at $56^{\circ} \mathrm{C}$ or $70^{\circ} \mathrm{C}$ (17). We investigated if moderate heating at $56^{\circ} \mathrm{C}$ or $70^{\circ} \mathrm{C}$ increases the LoB in G>A SNV dPCR assays. As contaminating RNA might serve as an additional template for dPCR assays, we also evaluated the effect of RNase A treatment on the number of target DNA molecules measured by dPCR. Using KRAS G12D assays, we found no substantial difference in LoB when whole blood genomic DNA was extracted with protease incubation at room temperature, $56^{\circ} \mathrm{C}$, or $70^{\circ} \mathrm{C}$, and this was not affected by the optional RNase $\mathrm{A}$ treatment during DNA elution (Tables 1 and 2). A previous study suggested that cytosine deamination was 15 times slower at $70^{\circ} \mathrm{C}$ than at $95^{\circ} \mathrm{C}$ (15), which might explain why heating at moderate temperatures did not show a measurable mutagenic effect. We also found that efficiency of target DNA recovery by dPCR ranged from $50.9 \%$ to $65.1 \%$, was similar at the 3 temperatures tested, and was not substantially influenced by RNase A treatment (Tables 1 and 2). The fact that the efficiency was not closer to $100 \%$ could be due to an overestimation of DNA concentration as measured by the NanoDrop ND-1000 spectrophotometer, which can be subject to absorption at $260 \mathrm{~nm}$ by co-purified impurities in the DNA sample (18). For a subset of samples, we re-measured concentrations using the Qubit assay and re-calculated targeted recovery efficiencies, which ranged from $68.3 \%$
Table 2. Limit of blank (LoB) of KRAS G12D (c.35G>A) droplet digital PCR (dPCR) assay using whole blood genomic DNA template isolated with RNase treatment.

\begin{tabular}{|c|c|c|c|c|c|c|}
\hline \multirow{2}{*}{ Treated with RNase A } & \multicolumn{3}{|c|}{ KRAS G12D false positive\% } & \multicolumn{3}{|c|}{$\%$ efficiency of WT allele recovery } \\
\hline & RT & $56^{\circ} \mathrm{C}$ & $70^{\circ} \mathrm{C}$ & RT & $56^{\circ} \mathrm{C}$ & $70^{\circ} \mathrm{C}$ \\
\hline Extraction 1_replicate 1 & 0 & 0 & 0 & $68.8 \%$ & $65.2 \%$ & $63.6 \%$ \\
\hline Extraction 1_replicate 2 & 0 & $0.00093 \%$ & 0 & $69.5 \%$ & $65.8 \%$ & $64.0 \%$ \\
\hline Extraction 2_replicate 1 & 0 & 0 & 0 & $64.1 \%$ & $61.6 \%$ & $56.8 \%$ \\
\hline Extraction 2_replicate 2 & $0.00113 \%$ & $0.00117 \%$ & $0.00231 \%$ & $57.9 \%$ & $57.7 \%$ & $58.4 \%$ \\
\hline Mean $(n=4)$ & $0.00028 \%$ & $0.00052 \%$ & $0.00058 \%$ & $65.1 \%$ & $62.5 \%$ & $60.7 \%$ \\
\hline SD & $0.00057 \%$ & $0.00061 \%$ & $0.00115 \%$ & $5.34 \%$ & $3.74 \%$ & $3.66 \%$ \\
\hline LoB & $0.00122 \%$ & $0.00153 \%$ & $0.00247 \%$ & & & \\
\hline
\end{tabular}

to $85.9 \%$ (Supplementary Tables S1 and S2). Although dPCR is more resistant to PCR inhibitors than real-time PCR methods, there is nevertheless some degree of inhibition in their presence (19). It is possible that co-purifying PCR inhibitors such as heme from whole blood (20) might also contribute in part to reducing target recovery efficiency.

Here we demonstrated that heatbased DNA fragmentation caused DNA mutagenesis that confounded SNV analysis of $\mathrm{G}>\mathrm{A}$ mutations in dPCR. Restriction enzyme digestion is an alternative DNA fragmentation approach that performed similarly to Covaris acoustic shearing. We should note that a limitation of our study is that we used spiked-in synthetic DNA of 200 or 225 bp in length, as opposed to fragmented genomic DNA. In future studies, cell line DNA harboring specific mutations could be used as an alternative. In conclusion, our study provides a rationale for choosing an optimal DNA fragmentation method for specific assay types and applications.

\section{Author contributions}

Q.K. and M.T. conceived the project. Q.K., B.P., and M.G. designed the experiments. Q.K. performed the experiments and analyzed the data. Q.K. and M.T. wrote the manuscript with contributions from all authors.

\section{Acknowledgments}

We thank William Kiffmeyer (RainDance Technologies) for advice on assay design, Andrew Rhim for use of the RainDrop dPCR platform, and Scott Tomlins for use of the Agilent TapeStation instrument. We also thank the DNA Sequencing Core Facility at the University of Michigan for use of their services. This project was supported by NIH R01DK085714 awarded to M.T. and UL1TR000433 from the National Center for Advancing Translational Sciences Postdoctoral Translational Scholar Program Fellowship to awarded to Q.K. The content is solely the responsibility of the authors and does not necessarily represent the official views of NCATS or the National Institutes of Health. This paper is subject to the NIH Public Access Policy.

\section{Competing interests}

The authors declare no competing interests. 


\section{References}

1. Simmonds, P., P. Balfe, C.A. Ludlam, J.O. Bishop, and A.J. Brown. 1990. Analysis of sequence diversity in hypervariable regions of the external glycoprotein of human immunodeficiency virus type 1. J. Virol. 64:5840-5850.

2. Sykes, P.J., S.H. Neoh, M.J. Brisco, E. Hughes, J. Condon, and A.A. Morley. 1992. Quantitation of targets for PCR by use of limiting dilution. Biotechniques 13:444-449.

3. Vogelstein, B. and K.W. Kinzler. 1999. Digital PCR. Proc. Natl. Acad. Sci. USA 96:9236-9241.

4. Hindson, B.J., K.D. Ness, D.A. Masquelier, P. Belgrader, N.J. Heredia, A.J. Makarewicz, I.J. Bright, M.Y. Lucero, et al. 2011. High-throughput droplet digital PCR system for absolute quantitation of DNA copy number. Anal. Chem. 83:86048610.

5. Taly, V., D. Pekin, L. Benhaim, S.K. Kotsopoulos, D. Le Corre, X. Li, I. Atochin, D.R. Link, et al. 2013. Multiplex picodroplet digital PCR to detect KRAS mutations in circulating DNA from the plasma of colorectal cancer patients. Clin. Chem. 59:1722-1731.

6. Oxnard, G.R., C.P. Paweletz, Y. Kuang, S.L. Mach, A. O'Connell, M.M. Messineo, J.J. Luke, M. Butaney, et al. 2014. Noninvasive detection of response and resistance in EGFR-mutant lung cancer using quantitative next-generation genotyping of cell-free plasma DNA. Clin Cancer Res. 20:1698-1705

7. Nie, Z.H., M.S. Seo, S.Q. Xu, P.C. Lewis, M. Mok, E. Kumacheva, G.M. Whitesides, P. Garstecki, and H.A. Stone. 2008. Emulsification in a microfluidic flow-focusing device: effect of the viscosities of the liquids. Microfluid Nanofluid 5:585-594

8. Milbury, C.A., Q, Zhong, J. Lin, M. Wiliams, J. Olson, D.R. Link, and B. Hutchison. 2014. Determining lower limits of detection of digital PCR assays for cancer-related gene mutations. Biomol Detect Quantif. 1:8-22.

9. Wang, J. and B. McCord. 2011. The application of magnetic bead hybridization for the recovery and STR amplification of degraded and inhibited forensic DNA. Electrophoresis 32:1631-1638.

10. Knierim, E., B. Lucke, J.M. Schwarz, M. Schuelke, and D. Seelow. 2011. Systematic comparison of three methods for fragmentation of long-range PCR products for next generation sequencing. PLoS ONE 6:e28240.

11. Yukl, S.A., P. Kaiser, P. Kim, P.L. Li, and J.K Wong. 2014. Advantages of using the QIAshredder instead of restriction digestion to prepare DNA for droplet digital PCR. Biotechniques 56:194-196.

12. Yang, Y. and J. Hang. 2013. Fragmentation of genomic DNA using microwave irradiation. Journal of biomolecular techniques. J Biomol Tech. 24:98103.

13. Huggett, J.F., C.A. Foy, V. Benes, K. Emslie, J.A. Garson, R. Haynes, J. Hellemans, M. Kubista, et al. 2013. The digital MIQE guidelines: Minimum Information for Publication of Quantitative Digital PCR Experiments. Clin. Chem. 59:892-902.

14. Armbruster, D.A. and T. Pry. 2008. Limit of blank, limit of detection and limit of quantitation. Clin. Biochem. Rev. 29(Supp/ 1):S49-S52.

15. Lindahl, T. and B. Nyberg. 1974. Heat-induced deamination of cytosine residues in deoxyribonucleic acid. Biochemistry 13:3405-3410.
16. Bhat, S., J.L.H. McLaughlin, and K.R. Emslie. 2011. Effect of sustained elevated temperature prior to amplification on template copy number estimation using digital polymerase chain reaction Analyst. 136:724-732

17. Bosch, L.A. 2014. A Proposal for an Individu alized Pharmacogenetic-Guided Warfarin Dosage Regimen for Puerto Rican Patients Commencing Anticoagulation Therapy. J Pharmacogenomics Pharmacoproteomics. 5:pii:T-001.

18. Simbolo, M., M. Gottardi, V. Corbo, M. Fassan, A. Mafficini, G. Malpeli, R. Lawlor, and A. Scarpa. 2013. DNA Quantification Workflow for Next Generation Sequecning of Histopathological Samples. PLoS ONE 8:e62692.

19. Dingle, T.C., R.H. Sedlak, L. Cook, and K.R. Jerome. 2013. Tolerance of droplet-digital PCR vs real-time quantitative PCR to inhibitory substances. Clin. Chem. 59:1670-1672.

20. Akane, A., K. Matsubara, H. Nakamura, S. Takahashi, and K. Kimura. 1994. Identification of the heme compound copurified with deoxyribonucleic acid (DNA) from bloodstains, a major inhibitor of polymerase chain reaction (PCR) amplification. J. Forensic Sci. 39:362-372.

Received 12 September 2015; accepted 05 January 2016.

Address correspondence to Muneesh Tewari, Department of Internal Medicine, University of Michigan, 109 Zina Pitcher Place, 1502 BSRB, SPC 2200, Ann Arbor, Ml 48109.E-mail: mtewari@med.umich.edu

To purchase reprints of this article, contact: biotechniques@fosterprinting.com

\section{The BMG LABTECH All Stars}

\section{Innovative, high-performance microplate readers for all assay needs}

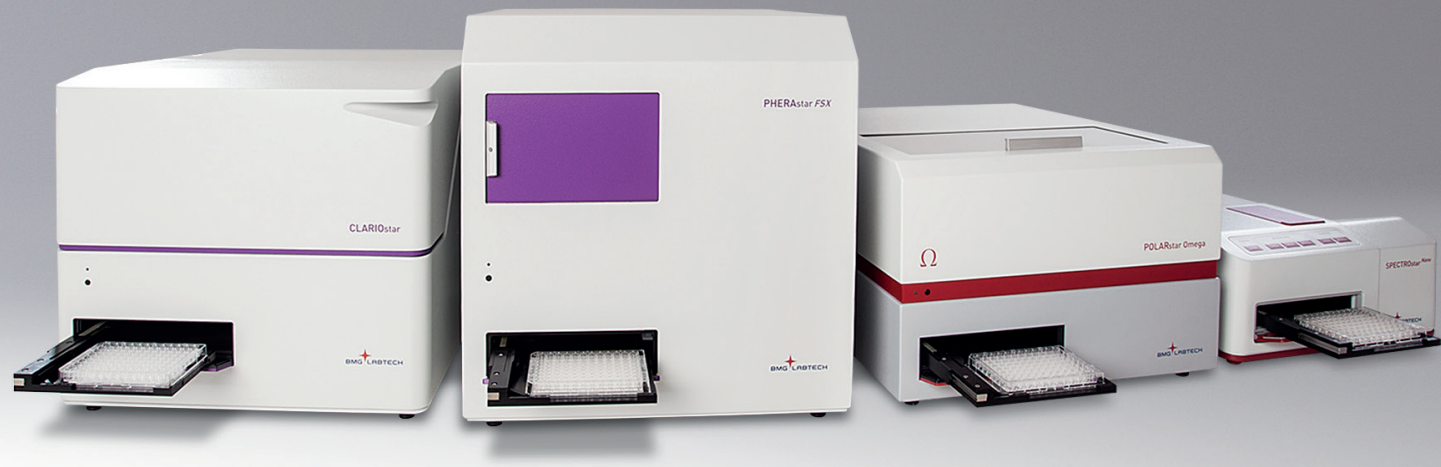

\section{CLARIOstar ${ }^{\circledast}$ \\ The most sensitive \\ monochromator-based \\ microplate reader.

\begin{abstract}
PHERAstar ${ }^{\circledR}$ FSX
The new gold standard for High Throughput Screening.
\end{abstract}

Omega series

Upgradable filter-based microplate readers for any

life science application.
SPECTROstar ${ }^{\circledR}$ Nano

Absorbance reader with ultra-fast detection of UV/Vis absorbance spectra. 